\title{
Chiral symmetry restoration in monolayer graphene induced by Kekulé distortion
}

\author{
Yasufumi Araki* \\ Department of Physics, The University of Tokyo, Tokyo 113-0033, Japan \\ E-mail: arakient.phys.s.u-tokyo.ac.jp
}

\begin{abstract}
We show a spontaneous breaking of the chiral symmetry and its restoration in monolayer graphene, by considering the system as a strongly coupled gauge theory. The chiral (sublattice) symmetry of monolayer graphene, which is spontaneously broken in the strong coupling limit of the Coulomb interaction, can be restored by introducing the Kekulé-patterned lattice distortion externally. We investigate such a behavior of the system analytically, by using the techniques of strong coupling expansion on the lattice gauge theory model, which preserves the original honeycomb lattice structure. We discuss the relation between the chiral phase transition and the spectral gap amplitude, and show the modification of the dispersion relation of the electrons/holes from that of non-interacting fermions.
\end{abstract}

The XXIX International Symposium on Lattice Field Theory - Lattice 2011

July 10-16, 2011

Squaw Valley, Lake Tahoe, California

${ }^{*}$ Speaker. 


\section{Introduction}

Graphene, a monoatomic layer material of carbon atoms, is one of the most intensely studied material both theoretically and experimentally, since its first experimental isolation in 2004 [1]. Due to the so-called "Dirac cone" structure of the dispersion relation of electrons and holes around half filling in the momentum space [2], monolayer graphene provides a physical realization of the (2+1)-dimensional massless Dirac fermion system [3], which attracts the field of particle physics as well. The electromagnetic field, on the other hand, still propagates in the (3+1)-dimensional space, so that the whole system can be effectively described by the "reduced quantum electrodynamics (QED)" [4].

The electron-electron interaction is supposed to play an important role in the monolayer graphene, since it remains unscreened around half filling and its effective strength is largely enhanced due to the discrepancy of the speed of light and the speed (Fermi velocity) of electrons and holes, if the system is suspended in vacuum. In reduced QED, such a strong Coulomb interaction can cause a spontaneous breaking of chiral symmetry, leading to the dynamical mass generation of the fermions. In graphene, the chiral symmetry of fermions corresponds to the inversion symmetry between two triangular sublattices of the honeycomb lattice. Since the above mechanism of gap generation is analogous to the dynamical quark mass generation mechanism in quantum chromodynamics (QCD), there have been several studies on the electron-electron interaction in graphene by employing the techniques in common with the analysis of QCD [5, 6].

There is another way to open a spectral gap in graphene without breaking the "chiral symmetry". The "Kekule distortion" [7], which is characterized by the alternating bond strength in the honeycomb lattice and is suggested to be introduced externally by the adatoms on the layer [8], opens a finite spectral gap proportional of the amplitude of the distortion. There have been few studies that consider both the spontaneous chiral symmetry breaking and the Kekule distortion, and their interplay at long wavelength, i.e. in the mean field, has not yet been understood in the previous works.

In this work, we consider the monolayer graphene system under an external Kekule distortion, and propose a chiral symmetry restoration mechanism induced by the lattice distortion. In order to consider the lattice distortion effect, we employ the effective model of the U(1) gauge theory, with the original honeycomb lattice structure. We consider the behavior of the system around the strong coupling limit of the Coulomb interaction by the technique of strong coupling expansion of lattice gauge theory, which is also employed in the QCD analysis, and observe the behavior of the spontaneous chiral symmetry breaking by varying the amplitude of the Kekule distortion. Finally, we show that the chiral symmetry is completely restored under a sufficiently large distortion amplitude, even in the strong coupling limit of the Coulomb interaction. The total magnitude of the spectral gap is calculated as a function of the Kekulé distortion amplitude.

\section{Lattice effective model}

In order to construct the model action of the system with the original honeycomb lattice struc- 
ture, we start from the conventional tight-binding Hamiltonian,

$$
H=-\sum_{\mathbf{r} \in A} \sum_{i=1,2,3}\left[h a^{\dagger}(\mathbf{r}) b\left(\mathbf{r}+\mathbf{s}_{i}\right)+\text { H.c. }\right] \text {. }
$$

which describes the hopping of an electron with amplitude $h$ between two nearest neighboring sites. Here $a\left(a^{\dagger}\right)$ and $b\left(b^{\dagger}\right)$ are the annihilation (creation) operators of electrons on the lattice cites in A and B sublattices respectively, and $\mathbf{s}_{i=1,2,3}$ are the hopping directions, with the lattice spacing $a=\left|\mathbf{s}_{i}\right|=1.42 \AA$. By diagonalizing this Hamiltonian in the momentum space, its energy eigenvalue shows the Dirac cone structure $E\left(\mathbf{K}_{ \pm}+\mathbf{k}\right)=h\left|\Phi\left(\mathbf{K}_{ \pm}+\mathbf{k}\right)\right|=v_{F}|\mathbf{k}|+O\left(k^{2}\right)$ around two Dirac points $\mathbf{K}_{ \pm}$, where $\Phi(\mathbf{k}) \equiv \sum_{i=1,2,3} e^{i \mathbf{k} \cdot \mathbf{s}_{i}}$. The Fermi velocity $v_{F}=(3 / 2) a h=3.02 \times 10^{-3}$ is considerably smaller than the speed of light. This Hamiltonian possesses an inversion symmetry between two sublattices $A$ and $B$, which can be extended to the continuous $\mathrm{U}(1)_{\mathrm{A}}$ symmetry in the low-energy region.

The external Kekule distortion is described by the additional hopping amplitude $\delta h$ with a spatial modulation,

$$
\delta h_{i}(\mathbf{r})=\frac{\Delta}{3}\left[e^{i\left(\mathbf{K}_{+} \cdot \mathbf{s}_{i}+\mathbf{G} \cdot \mathbf{r}\right)}+\text { c.c. }\right],
$$

where $\Delta$ is the amplitude of the distortion, and $\mathbf{G} \equiv \mathbf{K}_{+}-\mathbf{K}_{-}$. The Kekulé distortion does not break the sublattice symmetry, but it opens a spectral gap, $E\left(\mathbf{K}_{ \pm}+\mathbf{k}\right) \simeq v_{F} \sqrt{|\mathbf{k}|^{2}+\Delta^{2}}$. In the presence of the Kekule distortion, the translational symmetry by the sublattice spacing gets partially broken, so that the Brillouin zone $\Omega$ is split into three parts (see Fig,1).

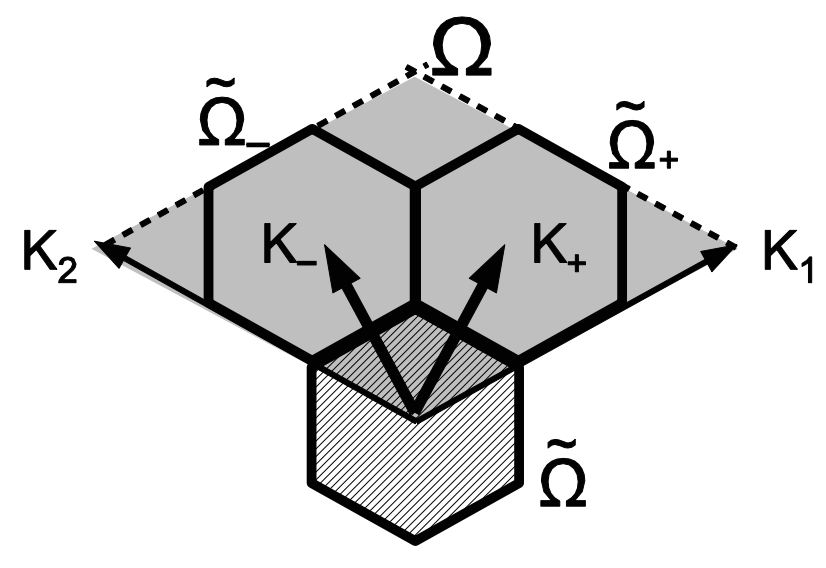

Figure 1: A schematic picture of the Brillouin zone corresponding to the honeycomb lattice. The Brillouin zone $\Omega$ (gray rhombic region) is spanned by the reciprocal lattice vectors $\mathbf{K}_{1}$ and $\mathbf{K}_{2}$. If the Kekulé distortion pattern [Eq.(2.2)] is introduced, $\Omega$ is split into three hexagonal cells: $\tilde{\Omega}$ and $\tilde{\Omega}_{ \pm}$, surrounding $\mathbf{k}=0$ and $\mathbf{K}_{ \pm}$ (Dirac points) respectively.

From the Hamiltonian in Eq. 2.17, the effective action for fermions $S_{F}$ is derived with the imaginary time $(\tau)$ formulation. Here we perform the temporal scale transformation $\tau \rightarrow \tau^{\prime} / v_{F}$, so that the Fermi velocity can be rescaled to be unity. The temporal direction is discretized with the lattice spacing $a_{\tau^{\prime}}\left(=v_{F} a_{\tau}\right)$ equal to the spatial lattice spacing $a$. This discretization generates a pair of fermion doublers, which we consider here as the spin (up/down) degrees of freedom. 
In this lattice model, the effect of the electromagnetic field is implemented by U(1) link variables between spatially or temporally neighboring sites. Due to the temporal scale transformation, the electromagnetic field becomes strongly coupled by the factor $v_{F}^{-1}$ in the temporal direction, while weakly coupled by the factor $v_{F}$ in the spatial direction. Therefore, by the saddle point approximation, we can set the spatial link variables to unity. This simplification means that the retardation (magnetic) effect of the electromagnetic field can be neglected due to the discrepancy between the Fermi velocity and the speed of light, which is referred to as "instantaneous approximation." With this approximation, the effective action of the system can be constructed:

$$
\begin{aligned}
S_{F}= & \frac{1}{2} \sum_{\mathbf{r} \in A ; \tau^{\prime}}\left[a^{\dagger}(x) U_{\tau^{\prime}}(x) a\left(x+a_{\tau^{\prime}}\right)-\text { H.c. }\right]+\frac{1}{2} \sum_{\mathbf{r} \in B ; \tau^{\prime}}\left[b^{\dagger}(x) U_{\tau^{\prime}}(x) b\left(x+a_{\tau^{\prime}}\right)-\text { H.c. }\right] \\
& +\frac{a_{\tau^{\prime}}}{v_{F}} \sum_{\mathbf{r} \in A, \tau^{\prime}} \sum_{i=1}^{3}\left[\left(h+\delta h_{i}(\mathbf{r})\right) a^{\dagger}(x) b\left(x+\mathbf{s}_{i}\right)+\text { H.c. }\right], \\
S_{G}= & \sqrt{3} \beta \sum_{\mathbf{r} \in A}\left[3-\frac{1}{2} \sum_{j=1,2,3}\left(U_{\tau^{\prime}}\left(\mathbf{r}+\mathbf{s}_{j}, \tau^{\prime}\right) U_{\tau^{\prime}}^{*}\left(\mathbf{r}, \tau^{\prime}\right)+\text { c.c. }\right)\right],
\end{aligned}
$$

where the position $x \equiv\left(\mathbf{r}, \tau^{\prime}\right)$, and the temporal link variable $U_{\tau^{\prime}}\left(\mathbf{r}, \tau^{\prime}\right)=\exp \left[i e \int_{\tau^{\prime}}^{\tau^{\prime}+a_{\tau^{\prime}}} d \tau^{\prime} A_{0}\right]$. The parameter $\beta \equiv v_{F} \varepsilon / e^{2}$ represents the inverse of the coupling strength, which is 0.037 in the vacuum-suspended graphene.

\section{Chiral symmetry breaking and restoration in the strong coupling limit}

Since $\beta$ is sufficiently small, we expect that the expansion around $\beta=0$ (strong coupling limit) works well. In this work, we only consider the leading order $\left[O\left(\beta^{0}\right)\right]$ in the strong coupling expansion, so that the gauge term $S_{G}$ does not contribute to the partition function:

$$
Z^{(0)}=\int\left[d \chi^{\dagger} d \chi\right][d U] e^{-S_{F}} \cdot \quad(\chi=a, b)
$$

By integrating out all the link variables, a contact 4-Fermi term $-(1 / 4) n_{\chi}(x) n_{\chi}\left(x+a_{\tau^{\prime}}\right)$ is generated, where $n_{\chi}(x)=\chi^{\dagger}(x) \chi(x)$ is the local charge density on the site $x$. Here we convert this term into fermion bilinear by Stratonovich-Hubbard transformation, with the bosonic auxiliary field $\sigma$ corresponding to the charge density difference between $\mathrm{A}$ and B sublattices, $\left\langle n_{a}-n_{b}\right\rangle . \sigma$ serves as the order parameter of the spontaneous sublattice symmetry breaking, which corresponds to the "chiral condensate" $\langle\bar{\psi} \psi\rangle$ of Dirac fermions, and it gives an effective mass term to the fermions.

The partition function of the system is given by integrating out the fermion degrees of freedom. Then we can obtain the effective potential per a pair of A and B sites, $F_{\text {eff }}^{(0)}(\sigma ; \Delta)=-\frac{1}{V N_{\tau^{\prime}}} \ln Z^{(0)}$, as a function of the auxiliary field $\sigma$ with the external parameter $\Delta$. When there is no Kekulé distortion, the effective potential is given as

$$
F_{\text {eff }}^{(0)}(\sigma)=\frac{1}{2} \sigma^{2}-\frac{1}{V} \int_{\mathbf{k} \in \Omega} d^{2} \mathbf{k} \ln \left[\left(\frac{\sigma}{2}\right)^{2}+\left|\frac{2}{3} \Phi(\mathbf{k})\right|^{2}\right],
$$

where the first term corresponds to the tree level of $\sigma$ and the second term comes from the oneloop effect of the fermion. Due to the interplay between these two terms, the effective potential 


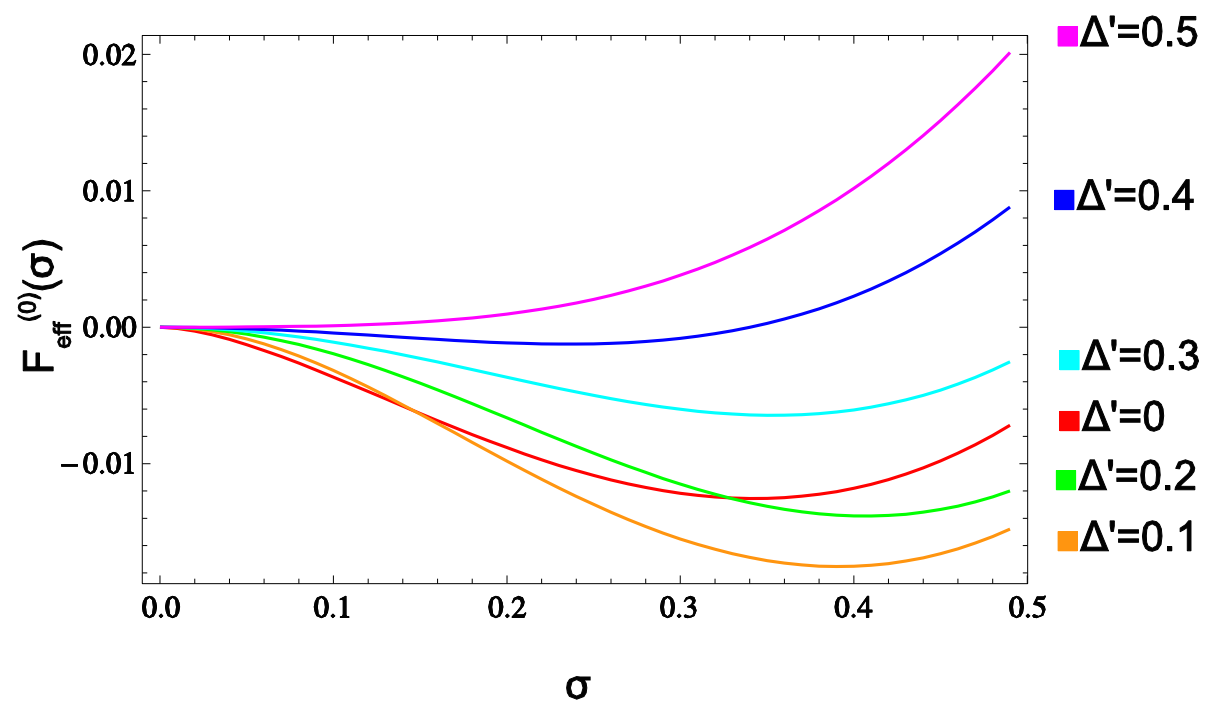

Figure 2: The behavior of the effective potential $F_{\text {eff }}^{(0)}(\sigma ; \Delta)$, with several values of the Kekule distortion $\Delta^{\prime}=\Delta / 3 h$. When $\Delta^{\prime}=0.5$, the effective potential becomes a monotonically increasing function.

has a minimum at finite $\sigma$, i.e. the sublattice symmetry of the honeycomb lattice is spontaneously broken at $\Delta=0$. Since the second term is dependent on the Kekule distortion $\Delta$, the effective potential changes its shape by varying the value of $\Delta$ (see Fig 2 ). Therefore, the expectation value of $\sigma$ behaves as a function of $\Delta$, as shown in Fig 3

The amplitude of the spotaneous sublattice (chiral) symmetry breaking, $\sigma(\Delta)$, quadratically grows around $\Delta=0$, while it eventually drops to zero for sufficiently large $\Delta$. The critical value of $\Delta$ is $\Delta_{C} / 3 h=0.485$, where $\sigma(\Delta)$ exhibits the second-order phase transition behavior. The suppression of $\sigma(\Delta)$ means that the chiral symmetry, which is spontaneously broken in the strong coupling limit, is restored by the external Kekule distortion. It can be qualitatively understood in terms of the effective field theory with the four-component Dirac fermion representation: the propagator of the Dirac fermion $\left(\mathbf{k} \cdot \gamma+\sigma / 2+\Delta \gamma_{3}\right)^{-1}$ is suppressed as $|\Delta| \rightarrow \infty$, leading to a reduction of the fermion one-loop effect in the effective potential. As a result, the tree level term $\left(\sigma^{2} / 2\right)$ dominates, and the effective potential becomes a monotonically increasing function of $\sigma$. On the other hand, the increase of $\sigma(\Delta)$ around $\Delta=0$ cannot be understood along the above description, since it originates from the discrepancy between the exact dispersion relation and the approximated Dirac cone structure far from the Dirac points. If the Dirac cone approximation is applied to the effective potential, the resulting $\sigma(\Delta)$ decreases monotonically around $\Delta=0$.

As a result of the interplay between the spontaneous sublattice (chiral) symmetry breaking and the external Kekule distortion, the amplitude of the spectral gap is also modified from that of the free fermions:

$$
E\left(\mathbf{K}_{ \pm} ; \Delta\right)=\sqrt{\left[v_{F} \sigma(\Delta) / 2 a_{\tau^{\prime}}\right]^{2}+\Delta^{2}}
$$

as a function of the distortion amplitude $\Delta$. When $\Delta<\Delta_{C}$, the gap amplitude is enhanced from that of the free fermions by the effect of sublattice symmetry breaking (effective mass term). When $\Delta>\Delta_{C}$, on the other hand, since the sublattice symmetry breaking is completely suppressed, the 


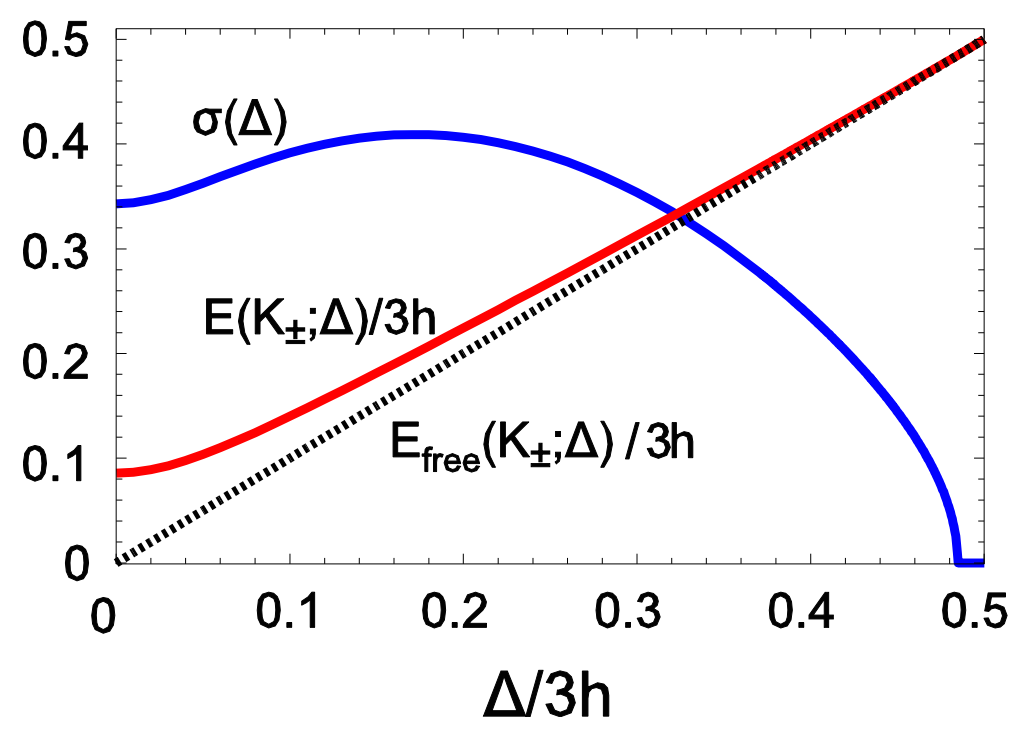

Figure 3: Charge density imbalance between two sublattices, $\sigma$, and the total energy gap $E\left(\mathbf{K}_{ \pm} ; \Delta\right)=$ $\sqrt{\left(v_{F} \sigma / 2 a_{\tau^{\prime}}\right)^{2}+\Delta^{2}}$, as the functions of the external Kekulé distortion $\Delta . \sigma(\Delta)$ first grows and then decreases as $|\Delta|$ increases, vanishing at the critical value $\Delta_{C} / 3 h=0.485$, while $E\left(\mathbf{K}_{ \pm} ; \Delta\right)$ remains finite for any value of $\Delta$. The dotted line shows the gap amplitude of the free fermion.

gap amplitude is not modified from that of the free fermions.

\section{Conclusion}

In this work, we have investigated the spontaneous sublattice (chiral) symmetry in monolayer graphene in the presence of the effectively strong Coulomb interaction. We have introduced the $\mathrm{U}(1)$ gauge theory on the honeycomb lattice as an effective field theory of the system, and have observed the behavior of the system in the strong coupling limit of the Coulomb interaction by the techniques of strong coupling expansion. As a result, while the fermion obtains a finite mass gap due to the spontaneous sublattice (chiral) symmetry breaking, we have found that the sublattice symmetry can be restored by the externally introduced Kekule distortion, which does not break the sublattice symmetry explicitly. Qualitatively, it can be understood as a flattening of the charge density distribution due to the strong hopping effect in the Kekulé distortion. As a consequence of such an interplay between the sublattice symmetry breaking and the Kekule distortion, the spectral gap of the fermion gets modified from that of the free fermions, so that it becomes no longer proportional to the Kekule distortion amplitude. We expect that such a behavior of the gap can have some effect on the gap engineering of graphene towards the application as electronic devices.

While only the leading order in the strong coupling expansion has been considered in this work, higher order terms have to be included in the realistic system. The next-to-leading order $[O(\beta)]$ terms consist of the interaction between nearest neighboring $(\mathrm{NN})$ sites,

$$
S_{F}^{(1)}=\frac{\sqrt{3} \beta}{8} \sum_{\mathbf{r}, \tau^{\prime}} \sum_{i=1,2,3}\left[a^{\dagger}(x) b\left(x+\mathbf{s}_{i}\right) b^{\dagger}\left(x+\mathbf{s}_{i}+a_{\tau^{\prime}}\right) a\left(x+a_{\tau^{\prime}}\right)+\text { H.c. }\right],
$$


which may effectively modify the NN hopping strength. Therefore, it may either renormalize the Fermi velocity $v_{F}$, or cause a spontaneous Kekulé distortion. With a sufficiently strong NN interaction, our preliminary study shows a first-order phase transition between the sublattice symmetrybroken phase and the spontaneous Kekulé distortion phase [9] 10]. Moreover, if the phononmediated interaction is taken into account, there may also appear a BCS-type (superconducting) order [11]. Thus, including various interaction terms, we expect that a rich phase structure of monolayer graphene can be seen by performing the strong coupling expansion order by order.

\section{Acknowledgments}

The author thanks H. Aoki, C. Detar, J. Drut, T. Hatsuda, K. Nomura and S. Sasaki for valuable comments and discussions. This work is supported by a Grant-in-Aid for the Japan Society for the Promotion of Science (DC1, No.22.8037).

\section{References}

[1] K. S. Novoselov et al., Science 306, 666 (2004).

[2] P. E. Wallace, Phys. Rev. 71, 622 (1947).

[3] G. W. Semenoff, Phys. Rev. Lett. 53, 2449 (1984).

[4] E. V. Gorbar, V. P. Gusynin and V. A. Miransky, Phys. Rev. D 64, 105028 (2001).

[5] Reviewed in A. H. Castro Neto, Physics 2, 30 (2009).

[6] Y. Araki and T. Hatsuda, Phys. Rev. B 82, 121403(R) (2010); Y. Araki, Annals Phys. (N. Y.) 326, 1408 (2011).

[7] N. A. Viet, H. Ajiki and T. Ando, J. Phys. Soc. Jpn. 63, 3036 (1994).

[8] M. Farjam and H. Rafii-Tabar, Phys. Rev. B 79, 045417 (2009).

[9] S. Raghu, X.-L. Qi, C. Honerkamp and S.-C. Zhang, Phys. Rev. Lett. 100, 156401 (2008).

[10] C. Weeks and M. Franz, Phys. Rev. B 81, 085105 (2010).

[11] L. H. C. M. Nunes, R. L. S. Farias and E. C. Marino, arXiv:1010.4279 [cond-mat.mes-hall]. 